\title{
Synthesis of Pure Silica MFI Zeolites Using Imidazolium-based Long Dications. A Comparative Study of Structure-directing Effects Derived from a Further Spacer Length Increase
}

\author{
Peng Lu, ${ }^{\text {abc }}$ Luis Gómez-Hortigüela ${ }^{d}$ and Miguel A. Camblor*a
}

Length-dependent structure direction of linear methylimidazolium-based dications towards the MFI zeolite, previously known only for the tetramethylene spacer case, has been found as well for the octamethylene and decamethylene spacers. This only works in highly concentrated conditions, while dilution always tends to favor TON, a default structure that is the only zeolite obtained with the other dications reported (with tri-, penta- and hexamethylene spacers). The location and conformation of the dications have been studied by molecular mechanics simulations. As the longer dications introduce a lower density of positive charges in the zeolite, their density of connectivity defects also decreases. Finally, these long dications cannot easily place each charged imidazolium ring in the two possible orientations found for the tetramethylene case (either parallel or perpendicular to the $4 \mathrm{MR}$ close to which $\mathrm{F}^{-}$sites). Hence, while the three MFI materials display two ${ }^{19} \mathrm{~F}$ NMR resonances at similar chemical shifts, their relative intensities strongly vary as a function of the spacer length.

\section{Introduction}

High silica zeolites are generally prepared by making use of the so-called structure-directing effects. Among these, structure direction by organic cations and neutral molecules (organic structure-directing agents, SDA) are of particular interest due to the large number of zeolites they have afforded. While their structure-directing action may vary widely from a loose space-filling to a strong and specific "templating", ${ }^{1}$ several characteristics have been deemed important in determining their action (size, flexibility, $\mathrm{C} / \mathrm{N}$ ratio,....). ${ }^{2}$ One aspect that perhaps has received not enough attention so far and until very recently is the distance between positive charges in the case of linear dications and polycations. Linear SDAs with more than one charge per entity were first introduced by Daniels et al., ${ }^{3}$ and subsequently studied also by others. ${ }^{4-7}$ Of particular interest was the observation of size effects, i.e., distinct structure-direction related to specific charge-to-charge separation lengths. ${ }^{5-7}$ This was also the case of our report on pure silica zeolite synthesis using dications based on two methylimidazolium units separated by polymethylene linkers of varying length ( $n=3,4,5,6$ methylene units), where MFI emerged only at $n=4$ over an otherwise featureless crystallization landscape merely yielding the "default structure" TON. ${ }^{8}$ We apply the term "default structures" to zeolites whose frameworks are rather stable on their own and which, not demanding a strong and specific structure-direction, may crystallize when direction lacks specificity. Size-effects have recently gained appeal after dications have provided a strategy for the synthesis of enantiomerically-enriched STW zeolites. ${ }^{9}$

In this work, we build on our prior report, ${ }^{8}$ to show that for methylimidazolium-based dications, MFI emerged again when the length of the spacer approximately doubles (i.e., for $n=8$ and 10).

\section{Experimental Section}

\section{Synthesis of the organic structure-directing agents}

The imidazolium-based dications used in this work were synthesized by reacting 1-methylimidazole with the corresponding linear $\alpha, \omega$-dibromo-n-alkane with $n=8$ and 10 carbon atoms, forming a doubly-charged cation with the imidazolium moieties on each end of the methylene chain, denoted as $8 \mathrm{BI}$ and $10 \mathrm{BI}$, respectively. A typical synthesis route is as follows. $0.14 \mathrm{~mol}(11.610 \mathrm{~g})$ of 1-methylimidazole (Sigma-Aldrich, 99\%) was dissolved in $50 \mathrm{ml}$ chloroform (Sigma-Aldrich, 99\%) in a flask under magnetic stirring until completely dissolved, then, $0.07 \mathrm{~mol}$ of the corresponding linear alkyl dibromide (Sigma-Aldrich, $98 \%$ for $n=8$, and $97 \%$ for $n=10$ ) was added followed by another $50 \mathrm{ml}$ of chloroform (Sigma-Aldrich, 99\%). The above mixture was refluxed for 4 days and the target bromides were obtained after the solvent was removed by rotary evaporation.

${ }^{1} \mathrm{H}$ and ${ }^{13} \mathrm{C}$ NMR spectra of the bromides dissolved in $\mathrm{D}_{2} \mathrm{O}$ confirmed that the products synthesized were $8 \mathrm{BI}$ and $10 \mathrm{BI}$ (Figure $\mathrm{S} 1$ and 1) with acceptable purity. The bromide salts were converted into their corresponding hydroxide form by anion exchange. Dowex anion exchange resin 550A (OH) (Sigma-Aldrich, 1.1 mequiv./1 ml) was washed with deionized water several times until no ammonia smell, then, the dibromide salts were added to the resin-water mixture until dissolution and the mixture was kept under stirring overnight. The hydroxide solution was recovered by filtration and washing and was subsequently concentrated using rotoevaporation under vacuum at $323 \mathrm{~K}$. The final concentration of the hydroxide solution was determined by titration with hydrochloric acid $(0.1 \mathrm{~N})$ using phenolphthalein as indicator.

Synthesis of zeolites 
Tetraethylorthosilicate (TEOS, 98\%, Sigma-Aldrich) was mixed with the hydroxide aqueous solution of the SDA. The mixture was magnetically stirred at room temperature overnight and then heated until all the ethanol, produced from TEOS hydrolysis, completely evaporated and the desired water/silica ratio was reached. In some cases, extra water was added to compensate for a too low water/silica ratio. Hydrofluoric acid (HF, Sigma-Aldrich, 48\%) was then added while homogenizing with a spatula by hand for $15 \mathrm{~min}$. The resulting gel was transferred to Teflon liners inside stainless steel autoclaves which were heated at different temperatures while tumbling at $60 \mathrm{rpm}$. The autoclaves were taken out from the oven at various time intervals and the solid product was washed with ample amount of deionized water and dried at $100{ }^{\circ} \mathrm{C}$. The final composition of the reaction gel was 1 $\mathrm{SiO}_{2}: 0.25 \mathrm{R}(\mathrm{OH})_{2}: 0.5 \mathrm{HF}: x \mathrm{H}_{2} \mathrm{O}$, where $x$ varies between 2 and 8 . The calcination of the as-made samples was performed in a muffle oven in air at $600{ }^{\circ} \mathrm{C}$ for $5 \mathrm{~h}$.

\section{Characterization}

The recovered solids were identified by powder X-ray diffraction (PXRD) collected in a Bruker D8 Advance diffractometer using Cu K $\alpha$ radiation $\left(\lambda=1.5418 \AA\right.$ ) in the $2 \theta$ region between $5^{\circ}$ and $45^{\circ}$. Field emission scanning electron micrographs (FE-SEM) were collected using an XL30 S-FEG microscope. Multinuclear magic angle spinning (MAS) NMR spectra $\left({ }^{19} \mathrm{~F},{ }^{13} \mathrm{C}\right.$ and ${ }^{29} \mathrm{Si}$ MAS NMR) of as-synthesized samples were obtained at room temperature on a Bruker AV-400-WB equipment and the details have been given elsewhere. ${ }^{10} \mathrm{CHN}$ elemental analyses were carried out using a LECO CHNS-932 analyzer. Thermal gravimetric analyses were performed in a SDT Q600 TA instrument under air flow $\left(100 \mathrm{ml} / \mathrm{min}\right.$ ) heating from $25{ }^{\circ} \mathrm{C}$ to $1000{ }^{\circ} \mathrm{C}$ (with a heating rate of 10 ${ }^{\circ} \mathrm{C} / \mathrm{min}$ ). Nitrogen adsorption/desorption were carried out in a Micromeritics ASAP 2010 equipment at the $\mathrm{N}_{2}$ liquefaction temperature of $77 \mathrm{~K}$. The samples were outgassed under vacuum at $120^{\circ} \mathrm{C}$.

\section{Computational details}

Following our previous work, ${ }^{8}$ molecular mechanics simulations were performed in order to find and compare the location of $8 \mathrm{BI}$ and $10 \mathrm{BI}$ within the MFI framework; simulations were carried out using the Forcite module implemented in Materials Studio. ${ }^{11}$ The geometry of the MFI structure was kept fixed during the calculations. Molecular structures and the interaction energies of the organic SDA cations with the framework were described with the CVFF forcefield. ${ }^{12}$ The atomic charges for the organic cations were calculated by the charge-equilibration method, ${ }^{13}$ setting the total net charge to +2 . The positive charge of the organic SDA dications was compensated by the framework by reducing the atomic charge of Si from 0.6 until neutrality. Framework oxygen charges were kept fixed to -0.3 .

Due to the large size of the SDA cations, $2 \times 2 \times 1 \mathrm{MFI}$ supercells have been used as the zeolite model. The molecules were manually docked in the framework in the required position/orientation. The most stable location for the SDA cations was then obtained by simulated annealing. The interaction energy was calculated by subtracting the energy of the dications in vacuo to the total energy of the system; all the energy values are given in $\mathrm{kcal} / \mathrm{mol}$ of SDA.

\section{Results and Discussion}

Zeolite Synthesis

Table 1 Synthesis results of pure silica zeolites using $8 \mathrm{BI}$ and $10 \mathrm{BI}$ as SDAs

\begin{tabular}{|c|c|c|c|c|}
\hline SDA & temperature $\left({ }^{\circ} \mathrm{C}\right)$ & $\mathrm{H}_{2} \mathrm{O} / \mathrm{SiO}_{2}$ & time (day) & phase \\
\hline \multirow[t]{9}{*}{$8 \mathrm{BI}$} & 175 & 2.6 & 1 & $\mathrm{MFI}$ \\
\hline & & & 3 & $\mathrm{MFI}$ \\
\hline & & & 9 & MFI \\
\hline & & 4.0 & 1 & MFI \\
\hline & & & 3 & MFI \\
\hline & & & 7 & $\mathrm{MFI}$ \\
\hline & & 8.0 & 1 & TON+AM \\
\hline & & & 3 & TON+unknown \\
\hline & & & 5 & TON(unknown) \\
\hline \multirow[t]{12}{*}{$10 \mathrm{BI}$} & 150 & 3.0 & 1 & AM \\
\hline & & & 4 & MFI \\
\hline & & & 10 & MFI \\
\hline & 175 & 3.2 & 1 & $\mathrm{MFI}+\mathrm{AM}$ \\
\hline & & & 3 & MFI \\
\hline & & & 7 & MFI \\
\hline & & 4.2 & 1 & $\operatorname{MFI}(A M)$ \\
\hline & & & 3 & MFI \\
\hline & & & 7 & MFI \\
\hline & & 8.0 & 1 & $\mathrm{AM}(\mathrm{MFI})$ \\
\hline & & & 4 & MFI(TON) \\
\hline & & & 7 & TON \\
\hline
\end{tabular}



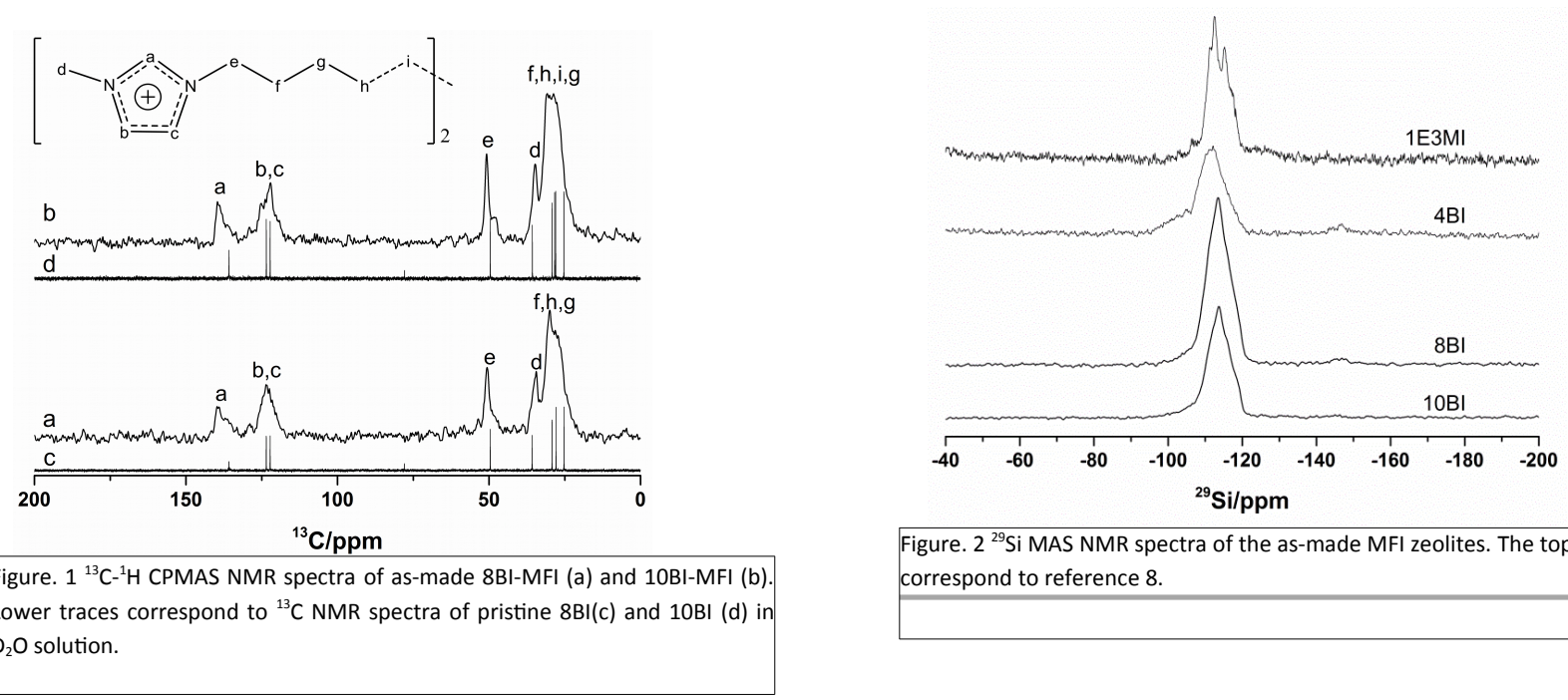

Figure. $2{ }^{29} \mathrm{Si}$ MAS NMR spectra of the as-made MFI zeolites. The top two traces correspond to reference 8 .

The synthesis parameters and phases obtained are summarized in Table 1. The two SDAs used in this work both showed a strong inclination towards $\mathrm{MFI}$, in sharp contrast with our previous report that bis(methylimidazolium) cations with 3-6 methylene spacers consistently produced the "default zeolite" TON except for 4BI which showed a loose structure direction effect towards MFI, which subsequently transformed to TON by Oswald ripening. ${ }^{5,8}$ The two SDAs, $8 \mathrm{BI}$ and 10BI, both demonstrated strong specificity towards MFI under concentrated conditions (water/silica ratios below 4.2) and no sign of transformation to TON was observed even after prolonged heating (the maximum crystallization time tested was 9 days for $8 \mathrm{BI}$ and 10 days for $10 \mathrm{BI}$; the MFI-framework was confirmed by XRD patterns shown in Figure S2). However, this specificity was weakened when working under more diluted conditions (water/silica ratios equal to 8.0 for both SDAs), in agreement with the Villaescusa's rule, ${ }^{14}$ which states that less dense phases (in this case MFI) tend to be more favored at higher concentrations. ${ }^{15}$ At those dilutions, TON was the crystalline phase obtained for $8 \mathrm{BI}$, though with impurities such as amorphous and unidentified phases (Figure S3). In contrast, 10BI showed a sign of MFI under short heating time (1 day) and TON appeared as a minor phase after prolonged heating and finally became pure with Oswald ripening (Figure S4). These different behaviors under high water content suggest a higher structure-directing ability to MFI and also a higher stabilization ability against transformation of $10 \mathrm{BI}$ over $8 \mathrm{BI}$, and thus, the preference for MFI can be summarized in combination with $4 \mathrm{BI}$ as: $10 \mathrm{BI}>8 \mathrm{BI}>4 \mathrm{BI}$. We recall again that in our prior work we got no hint of MFI when the number of carbon atoms in the linker was $n=3,5$ or 6 .

\section{Zeolite Characterization}

The chemical composition results of the as-made zeolites obtained in this work are listed in Table 2 . The $\mathrm{C} / \mathrm{N}$ ratios of the asmade zeolites are close to those of the pristine SDAs, suggesting that the SDAs are roughly preserved intact in the as-made zeolites, although there are small deviations that may raise doubt about this conclusion. The ${ }^{13} \mathrm{C}$ MAS NMR spectra (Figure 1 ), however, unambiguously confirmed the organics are predominantly intact, showing the expected resonances in the range of the alkyl $(0-60 \mathrm{ppm})$ and aromatic $(120-150 \mathrm{ppm})$ carbons (the ${ }^{13} \mathrm{C}$ NMR for pristine dications are shown as the lower sharp traces in Figure 1). There are around 2.7 and 2.6 organic dications per unit cell of $96 \mathrm{SiO}_{2}$ for the multidimensional medium pore MFI zeolites synthesized with $8 \mathrm{BI}$ and $10 \mathrm{BI}$, respectively, based on the measured $\mathrm{N}$ weight percentages (Table 2). Large deviations of the measured $\mathrm{H} / \mathrm{N}$ ratios from the theoretical ones were observed, and we deem likely that this might be caused by adsorbed water, by the presence of $\mathrm{Si}-\mathrm{OH}$ defects or both. With regard to $10 \mathrm{BI}-\mathrm{TON}$, it occludes a significantly smaller amount of $10 \mathrm{BI}$ dication than $10 \mathrm{BI}-\mathrm{MFI}\left(0.4\right.$ per cell of $24 \mathrm{SiO}_{2}$ or 1.6 per $96 \mathrm{SiO}_{2}$ ) as expected due to its unidimensional medium pore.

${ }^{29} \mathrm{Si}$ MAS NMR spectra of the as-made MFI zeolites synthesized in this work in comparison with our previously reported ones are shown in Figure 2. We include here MFI synthesized with the monocation 1-ethyl-3-methylimidazolium (1E3MI) because it can be formally considered a "monomer" of 4BI. ${ }^{8}$ All samples show the main resonances centered in the $-108 /-120$ ppm region assigned to the $\mathrm{Q}^{4} \mathrm{Si}$ species, i.e., Si coordinated in $\mathrm{Si}[\mathrm{OSi}]_{4}$ form. More interesting are the broad resonances in the region between -95/-

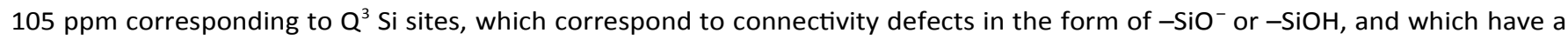
lower relative intensity for the materials synthesized with $1 \mathrm{E} 3 \mathrm{MI}, 8 \mathrm{BI}$ and $10 \mathrm{BI}$. The deconvolution of the ${ }^{29} \mathrm{Si} \mathrm{MAS} \mathrm{NMR}$ suggested that there are $2.0 \%, 16.6 \%, 5.0 \%$ and $4.7 \%$ of $\mathrm{Q}^{3}$ defects in as-made zeolites synthesized with $1 \mathrm{E} 3 \mathrm{MI}, 4 \mathrm{BI}, 8 \mathrm{BI}$ and $10 \mathrm{BI}$, respectively. Hence, the MFI zeolites synthesized with $8 \mathrm{BI}$ and $10 \mathrm{BI}$ contain a much lower density of defects than $4 \mathrm{BI}-\mathrm{MFI}$. The concentration of defects is clearly related to the organic contents: the maximum amount of fluoride in $\left[4^{1} 5^{2} 6^{2}\right]$ cages is 4 , so the excess positive charge of the cations needs to be balanced by defects. However, $\mathrm{SiO}^{-}$groups do not usually appear alone, they use to be associated to another $3 \mathrm{SiOH}$ so that the amount of $\mathrm{Q}^{3}$ is generally around 4 times larger than the amount of positive charges needed to be balanced. ${ }^{16}$ This is confirmed in our system: Table 3 summarizes the $Q^{3}$ data for the four as-made 
$\mathrm{MFI}$ zeolites (assuming there is as much $\mathrm{F}$ as possible in $\left[4^{1} 5^{2} 6^{2}\right]$ cages, i.e. 4 per unit cell of 96 tetrahedra). Indeed, the amount of defects derived from the

Table 2 Chemical composition of the as-made pure silica zeolites

\begin{tabular}{ccccccccc}
\hline SDA & Phase & $\mathrm{C}(\%)$ & $\mathrm{H}(\%)$ & $\mathrm{N}(\%)$ & $\mathrm{C} / \mathrm{N}^{\mathrm{a}}$ & $\mathrm{H} / \mathrm{N}^{\mathrm{a}}$ & $\mathrm{TG}^{\mathrm{b}}$ & empirical composition $^{\mathrm{c}}$ \\
\hline $8 \mathrm{BI}$ & $\mathrm{MFI}$ & 8.74 & 1.43 & 2.30 & $4.43(4.0)$ & $8.64(7.0)$ & $87.1(85.2)$ & $\left|\mathrm{C}_{16} \mathrm{H}_{28} \mathrm{~N}_{4} \mathrm{~F}_{2}\right|_{2.71}\left[\mathrm{SiO}_{2}\right]_{96}: 8.89 \mathrm{H}_{2} \mathrm{O}$ \\
$10 \mathrm{BI}$ & $\mathrm{TON}$ & 5.92 & 1.04 & 1.49 & $4.63(4.5)$ & $9.70(8.0)$ & $89.9(90.9)$ & $\left|\mathrm{C}_{18} \mathrm{H}_{32} \mathrm{~N}_{4} \mathrm{~F}_{2}\right|_{0.42}\left[\mathrm{SiO}_{2}\right]_{24}: 1.43 \mathrm{H}_{2} \mathrm{O}$ \\
$10 \mathrm{BI}$ & $\mathrm{MFI}$ & 9.16 & 1.48 & 2.21 & $4.83(4.5)$ & $9.31(8.0)$ & $85.8(86.5)$ & $\left|\mathrm{C}_{18} \mathrm{H}_{32} \mathrm{~N}_{4} \mathrm{~F}_{2}\right|_{2.63}\left[\mathrm{SiO}_{2}\right]_{96}: 6.89 \mathrm{H}_{2} \mathrm{O}$
\end{tabular}

${ }^{a}$ Molar ratios, the theoretical values are given in parentheses. ${ }^{b}$ Solid residues after thermal analysis to $1000{ }^{\circ} \mathrm{C}$ (the amount in parentheses stands for the $\mathrm{SiO}_{2}$ content in the unit cell given in the last column). 'SDA calculated from the $\mathrm{N}$ percent assuming the organics are intact, and charge-balanced by $\mathrm{F}^{-}$ion and that $\mathrm{SiO}_{2}$ is the residue after TG. The water was calculated from the excess $\mathrm{H} / \mathrm{N}$ ratio (it may be contributed by water or Si-OH defects or both).

\begin{tabular}{|c|c|c|c|c|}
\hline \multirow{2}{*}{ Parameter } & \multicolumn{4}{|c|}{ Sample } \\
\hline & 1E3MI-MFI & 4BI-MFI & 8BI-MFI & 10BI-MFI \\
\hline organic per u.c. & 4.4 & 3.9 & 2.7 & 2.6 \\
\hline excess positive charge & $4.4 \times 1-4=0.4$ & $3.9 \times 2-4=3.8$ & $2.7 \times 2-4=1.4$ & $2.6 \times 2-4=1.2$ \\
\hline $4 \times$ excess positive charge & 1.6 & 15.2 & 5.6 & 4.8 \\
\hline$Q^{3}(\%)$ & 2.0 & 16.6 & 5.0 & 4.7 \\
\hline$Q^{3} /$ u.c. & 2.0 & 15.9 & 4.8 & 4.5 \\
\hline$Q^{3} /(4 \times$ excess charge $)$ & 1.25 & 1.05 & 0.86 & 0.94 \\
\hline
\end{tabular}

${ }^{29}$ Si MAS NMR is about four times the expected excess positive charge.

Figure 3 shows the ${ }^{19} \mathrm{~F}$ MAS NMR spectra of the as-made MFI zeolites. The four samples show two similar resonances, with small but noticeable chemical shift differences with respect to each other. For the resonances at the lower field, the signals are centered at around $-65,-68,-69$ and $-70 \mathrm{ppm}$ for samples occluding cations of increasing size (Figure 3 ). In contrast, the higher field resonances are at around $-78 \mathrm{ppm}$ for the two shorter and at $-80 \mathrm{ppm}$ for the two longer cations. In addition, the relative intensities of these two resonances varied among different samples as shown in Table 4 . With regard to $n \mathrm{BI}-\mathrm{MFI}$ zeolites, the intensity of the low field resonance relative to the high field one increased along with the increase of the methylene chain (10BI$\mathrm{MFI}>8 \mathrm{BI}-\mathrm{MFI}>4 \mathrm{BI}-\mathrm{MFI})$. As for $1 \mathrm{E} 3 \mathrm{MI}-\mathrm{MFI}$, it gives the highest relative intensity of 10 to 1 , with the lower field resonance $(-65$ $\mathrm{ppm}$ ) much closer to the position of the one observed (-64 ppm) in MFI synthesized with TPA and F ions. ${ }^{17}$ As we reported earlier, the presence of the two resonances in $4 \mathrm{BI}-\mathrm{MFI}$ suggested two different chemical environments for the fluoride ion, which in turn

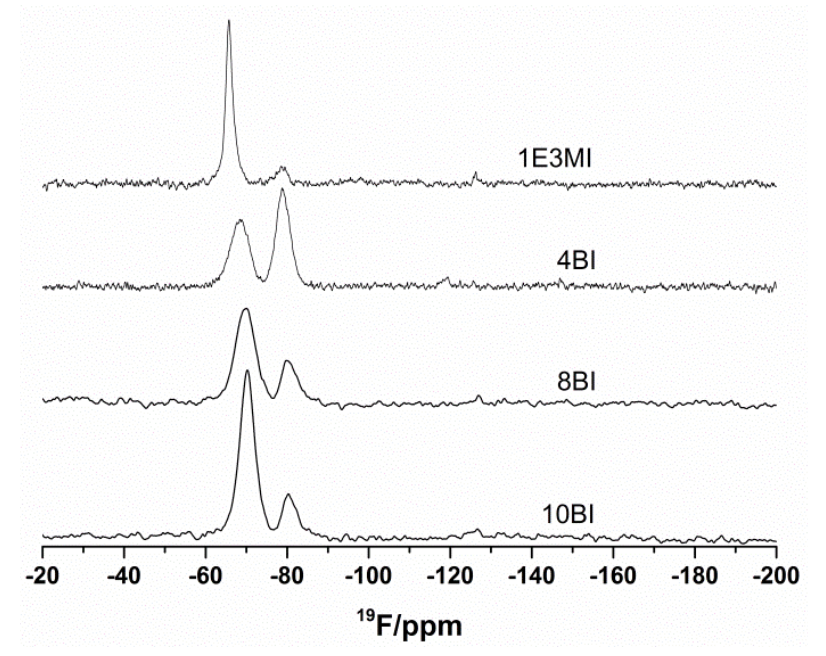

Figure. $3{ }^{19} \mathrm{~F}$ MAS NMR spectra of the as-made MFI zeolites.

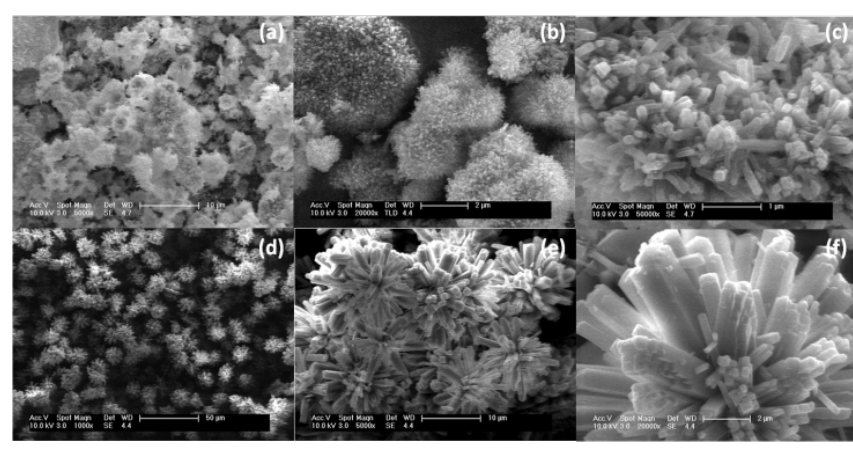

Figure. 4 SEM images of the as-made zeolites: 8BI-MFI (a, $b$ and c) and 10BI-MFI (d, e and f) synthesized at $175{ }^{\circ} \mathrm{C}$ with water/silica ratio of 4.0 and 4.2 , respectively. Note that magnifications at top and bottom are not the same.

should reflect different orientations of the imidazolium rings with regard to fluoride. ${ }^{8}$ Indeed, the DFT calculation results showed different interactions between the $\left[\mathrm{SiO}_{4 / 2} \mathrm{~F}\right]^{-}$units with the methyl group and the aromatic cloud of the imidazolium ring. This appears to be the case also for the longer dications reported here (see below). 
Table 4 The relative intensities of the ${ }^{19} \mathrm{~F}$ NMR resonances of the as-made MFI zeolites

\begin{tabular}{ccc}
\hline & & relative intensity \\
\cline { 2 - 3 } SDA & $\sim-70 \mathrm{ppm}$ & $\sim-80 \mathrm{ppm}$ \\
1E3MI & 10 & 1 \\
$4 \mathrm{BI}$ & 1 & 1 \\
8BI & 3 & 1 \\
$10 \mathrm{BI}$ & 4 & 1 \\
\hline
\end{tabular}

${ }^{1} \mathrm{H}$ MAS NMR spectra of MFI zeolites synthesized with the four different SDAs are shown in Figure S5. Compared with 1E3MI-MFI and $4 \mathrm{BI}-\mathrm{MFI}$, the new $8 \mathrm{BI}-\mathrm{MFI}$ and $10 \mathrm{BI}-\mathrm{MFI}$ zeolites synthesized in this work show poorly resolved spectra, probably due to the large molecule sizes and also different conformations in the channels. The broad resonance around $10.2 \mathrm{ppm}$ observed in 4BI$\mathrm{MFI}$, a proof of the existence of relatively strong $\mathrm{Si}-\mathrm{OH}$ hydrogen bonded to $\mathrm{Si}^{-} \mathrm{O}^{-}$groups, can not be distinguished in the other three MFI zeolites. ${ }^{18}$

The thermogravimetric analysis data of the as-made zeolites prepared here are shown in Figure S6. 10BI-TON showed almost no weight loss when heated up to $200{ }^{\circ} \mathrm{C}$ indicating the high hydrophobicity of the pure silica zeolite TON. The total weight loss up to $800{ }^{\circ} \mathrm{C}$ is $9.1 \%$, close to our reported value ( $\left.10 \%\right)$ in $n \mathrm{BI}-\mathrm{TON}(n=3-6)$, as expected for a mere unspecific pore filling. In contrast, $8 \mathrm{BI}-\mathrm{MFI}$ and $10 \mathrm{BI}-\mathrm{MFI}$ showed minor weight losses of $1.9 \%$ and $1.1 \%$ below $250{ }^{\circ} \mathrm{C}$, indicating probably more connectivity defects in the former than the later, and major ones in between $250{ }^{\circ} \mathrm{C}$ and $600{ }^{\circ} \mathrm{C}$, finally giving total weight losses of $14.7 \%$ and $13.7 \%$, respectively.

FField-emission SEM images of the as-made MFI zeolites are shown in Figure 4. The crystals of 8BI-MFI (Figure 4, (a), (b) and (c)) display smaller sizes than those of 10BI-MFI (Figure 4, (d), (e) and (f)), and both samples featured rod-like elongated morphologies. Interestingly, 10BI-MFI shows uniformly flower-like aggregates of crystal rods, which radiate from the center outwards, while a disordered arrangement of crystals into large non-uniform aggregates is exhibited for 8BI-MFI. Compared with the previously reported $4 \mathrm{BI}-\mathrm{MFI}{ }^{19}$ which showed a morphology with twin intergrowths of two plate-like crystals, one of the typical habits of MFI zeolites, the increase in length of the linker indeed caused a significant morphology variation. ${ }^{20}$

The porosity of calcined 8BI-MFI and 10BI-MFI has been characterized by $\mathrm{N}_{2}$ adsorption and desorption, which evidenced a hysteresis loop interpreted as due to interparticle mesoporosity (see SI Figure S7 and Table S1).

\section{Computational results}

Due to the non-commensurate packing value of the SDA cations within the MFI framework ( $\sim 2.6-2.7$ SDAs/u.c., and considering that there are two straight and two sinusoidal channels per MFI u.c.), we could only study the occlusion of one SDA dication on each type of location, i.e. neglecting packing (intermolecular) interactions, since no periodic arrangement of the SDAs with reasonable supercell size could be envisaged. We loaded 1 cation per $2 \times 2 \times 1 \mathrm{MFI}$ supercell, in the two types of channels, and initially with different relative orientations of the imidazolium rings (i.e. different molecular conformations): with both rings pointing to the same side, or to opposite sides. Then the most stable situations were found by simulated annealing. Table 5 reports the energy results of the stable locations of $8 \mathrm{BI}$ and $10 \mathrm{BI}$ in the two types of channels, and the stable configurations are shown in Figure $5(8 \mathrm{BI})$ and Figure $6(10 \mathrm{BI})$.

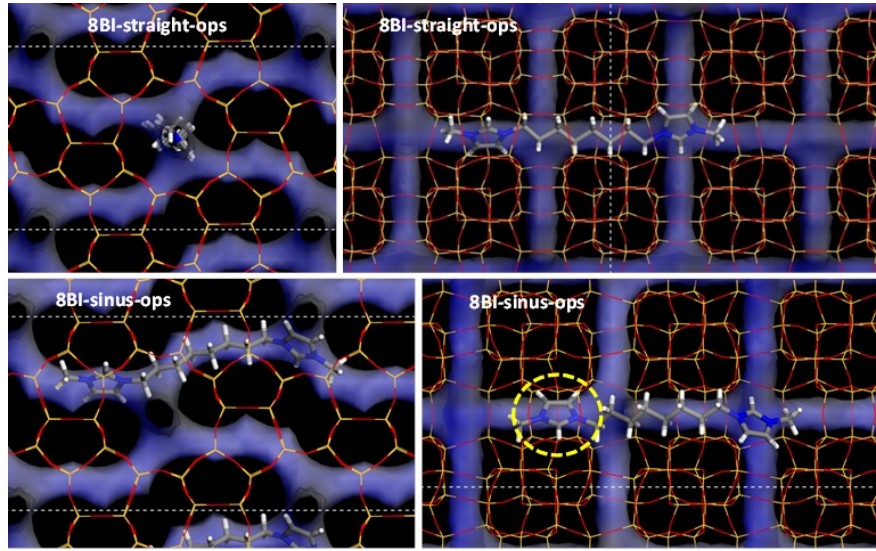

Figure. 5 Location of $8 \mathrm{BI}$ cations in the most stable configuration (without packing interactions) in straight (top) and sinusoidal (bottom) channels. 
Table 5 Relative energies (R.E., with respect to the most stable case for each dication) and interaction energies (I.E.) of the SDA cations located in the different channels and in different initial orientations; energies are given in $\mathrm{kcal} / \mathrm{mol}$ SDA.

\begin{tabular}{|c|c|c|c|c|}
\hline SDA & Channel & Conformation & R. E. & I. E. \\
\hline \multirow{4}{*}{$8 \mathrm{BI}$} & \multirow{2}{*}{ Straight [010] } & ss & 1.6 & -124.7 \\
\hline & & ops & 0.0 & -126.3 \\
\hline & \multirow{2}{*}{ Sinusoidal [100] } & ss & 7.4 & -118.9 \\
\hline & & ops & 4.8 & -121.5 \\
\hline \multirow{4}{*}{$10 \mathrm{BI}$} & \multirow{2}{*}{ Straight [010] } & ss & 0.5 & -132.4 \\
\hline & & ops & 0.0 & -132.9 \\
\hline & \multirow{2}{*}{ Sinusoidal [100] } & ss & 8.9 & -124.0 \\
\hline & & ops & 4.0 & -128.9 \\
\hline
\end{tabular}

Energy results clearly show that both cations fit better within the straight [010] channels (see relative energies in Table 5); moreover, in all cases, the molecular conformation with both imidazolium rings pointing to opposite sides (ops) is more stable (Figures 5 and 6). In the straight channels, due to the length of the cations, both $8 \mathrm{BI}$ and $10 \mathrm{BI}$ site with one ring in the channel intersections and the other between channel intersections (see Figures 5 and 6-top). Assuming a full-loading of these straight channels with the dications, this should give packing values of $\sim 1.70$ and 1.55 SDAs/u.c. for $8 \mathrm{BI}$ and $10 \mathrm{BI}$, respectively (calculated taking into account the projection along the ' $b$ ' direction of each dication, assuming a distance of $3 \AA$ between adjacent cations, and relating to the ' $b$ ' unit cell parameter); this packing value is clearly lower than the experimentally determined values of 2.6 and 2.7 SDAs/u.c., which indicates that the sinusoidal channels also have to be filled by the dications.



Figure. 6 Location of $10 \mathrm{BI}$ cations in the most stable configuration (without packing interactions) in straight (top) and sinusoidal (bottom) channels.

In the sinusoidal channels, the dications site with one of the imidazolium rings in-between channel intersections (close to the $\left[4^{1} 5^{2} 6^{2}\right]$ cages), and the other in the channel intersections (see Figures 5 and 6-bottom). The high density of single bonds enables the dications to follow the zig-zag direction of the sinusoidal channels. In this case, the energy difference between the two molecular conformations is higher, being clearly more stable the conformation with the rings pointing in opposite sides (Table 5). Other configurations where the dications are folded so that the two imidazolium rings sit in the two channels, with one ring in the sinusoidal and one in the straight channels with the spacer folded to follow the curvature (see Figure S8), were also studied and were found to be slightly less stable, with interaction energies of -118.9 and $-124.7 \mathrm{kcal} / \mathrm{mol}$ SDA for $8 \mathrm{BI}$ and $10 \mathrm{BI}$, respectively. These energies were only slightly smaller (by 2.6 and $4.2 \mathrm{kcal} / \mathrm{mol}$ for $8 \mathrm{BI}$ and $10 \mathrm{BI}$, respectively), than the most stable configuration in the sinusoidal channels, and hence this packing configuration could at some point take place if that favors the efficient space-filling of the MFI channels.

As previously mentioned, in order to reach the high packing values experimentally determined (2.7 and 2.6 SDAs/u.c. for 8BI and $10 \mathrm{BI}$, respectively), both types of channels have to be filled. Due to the large size of the SDA dications and the MFI unit cell dimensions, it is difficult to envisage a periodic arrangement of the organic dications within the MFI channels. It is expectable that the dications will fill the void space of both channel systems, possibly in the different conformations previously mentioned, in order to maximise the occupation of the channels. Taking into account the distance of the projection of the dications in the channels directions, we estimate an average distance between adjacent dications of $9 \AA$ and $8 \AA$ for $8 \mathrm{BI}$ and $10 \mathrm{BI}$, respectively, in 
order to fill the MFI channels with packing values of 2.7 and 2.6 SDAs/u.c. This means that the SDAs will fill both types of channels, though some free space will be available due to the non-commensurate sizes of the channels and the dications, with some dications located closer and some others more separate, depending on how the SDAs fit within the channel systems of MFI.

Regarding the different population of the ${ }^{19} \mathrm{~F}$ NMR signals, this could be tentatively explained by the different packing arrangement of these dications compared to $4 \mathrm{BI}$. As we observed in our previous work, ${ }^{8}$ the signal at $\sim-80 \mathrm{ppm}$ is caused by a special interaction of the $\mathrm{F}$ anions inside the $\left[4^{1} 5^{2} 6^{2}\right]$ cages with the imidazolium rings in a particular orientation (perpendicular to the $4 \mathrm{MR}$ of the $\left[4^{1} 5^{2} 6^{2}\right]$ cage); therefore, for this ${ }^{19} \mathrm{~F} \mathrm{NMR}$ signal to occur, one of the imidazolium rings of the dications has to site in that particular position, within the sinusoidal channels close to the $\left[4^{1} 5^{2} 6^{2}\right]$ cages (see dashed circles in Figures 5 and 6 bottom). The longer length of these dications implies a smaller amount of imidazolium rings that will site close to $\mathrm{F}$ in the required orientation to allow for this especial interaction, and hence the relative population of the ${ }^{19} \mathrm{~F}$ NMR signal should decrease with the length of the SDA dications, as is experimentally observed.

\section{Conclusion}

Bis-methylimidazolium dications of varying length neatly display size-dependent structure direction during the synthesis of pure silica zeolites in fluoride medium. While all of them may produce the default TON zeolite, dications with 4, 8 or 10 (but not 3, 5 or 6) carbons in the spacer are also able to produce MFI under concentrated conditions. We had previously demonstrated the commensurate effect in the case of the tetramethylene dication, and now we show again size-dependent structure-direction for the octamethylene and decamethylene dications that are around double in size. In the present cases, however, the amount of charges in the zeolite is significantly smaller and hence the materials present a much lower concentration of connectivity defects. Also, because of the longer length of these dications, the relative proportion of imidazolium rings oriented perpendicular to the $4 \mathrm{MR}$ of the $\left[4^{1} 5^{2} 6^{2}\right]$ cage, responsible for the ${ }^{19} \mathrm{~F}$ resonance at around $-80 \mathrm{ppm}$, is much smaller and, hence, the relative intensity of this signal is significantly smaller than in the tetramethylene dication case.

\section{Conflicts of interest}

There are no conflicts to declare.

\section{Acknowledgements}

Financial support by the Spanish Ministry of Economy and Competitiveness (projects MAT2015-71117-R and MAT2016-77496-R) is acknowledged. P.L. is grateful to the China Scholarship Council (CSC) for a fellowship and to the Dalian Institute of Chemical Physics for permission to leave. Centro Técnico Informático-CSIC is acknowledged for running the calculations, and Accelrys for providing the computational software.

\section{Notes and references}

1 M. E. Davis and R. F. Lobo, Chem. Mater., 1992, 4, 756-768.

2 Y. Kubota, M. M. Helmkamp, S. I. Zones and M. E. Davis, Microporous Materials, 1996, 6, 213-229.

3 R. H. Daniels, G. T. Kerr and L. D. Rollmann, J. Am. Chem. Soc., 1978, 100, 3097-3100.

4 M. E. Davis and C. Saldarriaga, J. Chem. Soc. Chem. Commun., 1988, 0, 920-921.

5 A. Moini, K. D. Schmitt, E. W. Valyocsik and R. F. Polomski, Zeolites, 1994, 14, 504-511.

6 S.-H. Lee, C.-H. Shin, D.-K. Yang, S.-D. Ahn, I.-S. Nam and S. B. Hong, Microporous Mesoporous Mater., 2004, 68, 97-104.

7 S. B. Hong, H.-K. Min, C.-H. Shin, P. A. Cox, S. J. Warrender and P. A. Wright, J. Am. Chem. Soc., 2007, 129, 10870-10885.

8 A. Rojas, L. Gomez-Hortiguela and M. A. Camblor, J. Am. Chem. Soc., 2012, 134, 3845-3856.

9 S. K. Brand, J. E. Schmidt, M. W. Deem, F. Daeyaert, Y. Ma, O. Terasaki, M. Orazov and M. E. Davis, Proc. Natl. Acad. Sci. U. S. A., 2017, 114, 5101-5106.

10 A. Rojas, E. Martinez-Morales, C. M. Zicovich-Wilson and M. A. Camblor, J. Am. Chem. Soc., 2012, 134, 2255-2263.

11 Forcite module. BIOVIA Materials Studio 2017 R2.

12 P. Dauber-Osguthorpe, V. A. Roberts, D. J. Osguthorpe, J. Wolff, M. Genest and A. T. Hagler, Proteins, 1988, 4, 31-47.

13 A. K. Rappe and W. A. Goddard, J. Phys. Chem., 1991, 95, 3358-3363.

14 S. B. H. Miguel A. Camblor, in Porous Materials, eds. D. W. Bruce, D. O'Hare, R. I. Walton, Willey, 2011, ch. 5.

15 M. A. Camblor, L. A. Villaescusa and M. J. Díaz-Cabañas, Top. Catal., 1999, 9, 59-76.

16 M. A. Camblor, P. A. Barrett, M. a.-J. Díaz-Cabañas, L. A. Villaescusa, M. Puche, T. Boix, E. Pérez and H. Koller, Microporous Mesoporous Mater., 2001, 48, 11-22.

17 C. A. Fyfe, D. H. Brouwer, A. R. Lewis and J.-M. Chézeau, J. Am. Chem. Soc., 2001, 123, 6882-6891.

18 H. Koller, R. F. Lobo, S. L. Burkett and M. E. Davis, J. Phys. Chem., 1995, 99, 12588-12596.

19 A. E. R. Núñez, PhD thesis, Universidad Autonoma de Madrid, 2012. 
20 G. Bonilla, I. Díaz, M. Tsapatsis, H.-K. Jeong, Y. Lee and D. G. Vlachos, Chem. Mater., 2004, 16, 5697-5705. 